\title{
Análisis morfométrico para la discriminación de especies: el caso del complejo Megalobulimus leucostoma
}

\author{
Morphometric analysis to discriminate between species: The case of the Megalobulimus leucostoma \\ complex
}

1 Departamento de Malacología y Carcinología Museo de Historia Natural - Universidad Nacional Mayor de San Marcos, Lima - Perú.

2 Laboratorio de Sistemática Molecular y Filogeografía - Facultad de Ciencias Biológicas - Universidad Nacional Mayor de San Marcos, Lima - Perú. Corresponding author: Victor Borda

Email Victor Borda: vicbp1@gmail.com Email Rina Ramirez: rina_rm@yahoo.com

Citación:

Borda V. \& R. Ramirez. 2014. Análisis morfométrico para la discriminación de especies: el caso de complejo Megalobulimus leucostoma. Revista peruana de biología 21(2): 117 - 124 (Octubre 2014). doi: http://dx.doi.org/10.15381/rpb.v21i2.9814

Fuentes de financiamiento:

El presente trabajo fue financiado por:

Beca Rufford Small Grants Foundation otorgada a Victor Borda.

Proyecto VRI-CSI101001091 de la Universidad Nacional Mayor de San Marcos otorgado a Rina Ramírez.

\section{Victor Borda $^{1,2}$ y Rina Ramirez ${ }^{1,2}$}

\begin{abstract}
Resumen
La plasticidad de los caracteres conquiliológicos ha conllevado a diversas descripciones erróneas y a la acumulación de sinonimias lo cual ha dificultado la discriminación entre especies. El género de moluscos terrestres Megalobulimus es un ejemplo de esta problemática. Dentro de éste se encuentra Megalobulimus leucostoma (Sowerby, 1835), que presenta tres subespecies las cuales no se logran diferenciar correctamente a partir de sus descripciones originales. El presente estudio busca discriminar a las subespecies de Megalobulimus leucostoma a partir del análisis morfométrico y de distribución. Ambos análisis establecen diferencias sustanciales entre M. I. leucostoma y M. I. lacunosus que no soportarían el estatus de subespecie de esta última. Megalobulimus leucostoma weyrauchi se ubica dentro de la gran gama de variabilidad conquiliológica de M. I. leucostoma y sus datos de distribución lo ubican en simpatría con la misma por lo cual su estatus como subespecie no estaría soportado, y podría considerarse como parte de M. I. leucostoma.
\end{abstract}

Palabras clave: Morfometría; Megalobuliminae; conchilla; moluscos terrestres.

\section{Abstract}

Plasticity of conchological characters had led to erroneous descriptions and the accumulation of synonyms making difficult the discrimination among species. The land snail genus Megalobulimus is an example of this problem. Megalobulimus leucostoma (Sowerby, 1835) has three subspecies which are difficult to differentiate by using the original descriptions. The aim of this paper is to discriminate among the subspecies of $M$. leucostoma by using morphometric and distribution analyses. Both provide substantial differences between M. I. leucostoma and M. I lacunosus that would not support the subspecies status of the former. Megalobulimus leucostoma weyrauchi fits into the great conchological variability of $M$. I. leucostoma; also the sympatric status between these two subspecies would not support the subspecies status of the former, and M. I. weyrauchi should be considered as part of M. I. leucostoma.

Keywords: morphometry; Megalobuliminae; shell; land mollusks.

\section{Introducción}

Uno de los problemas recurrentes en la discriminación de especies de moluscos es que la mayoría de sus descripciones están basadas en las características de las conchillas. Si bien existen casos en los que la diferenciación por caracteres conquiliológicos es eficiente, se considera que estos caracteres son afectados por evolución paralela o convergente en diferentes taxa (Suvorov 2002). La Subfamilia Megalobuliminae (Leme) (Familia Strophocheilidae) encaja dentro de esta problemática. Es una subfamilia de un único género, con cerca de 50 especies nominales y donde se encuentran los gasterópodos terrestres de mayor tamaño en la región neotropical (Bequaert 1948, Ramírez et al. 2003, Simone 2006), algunos de los cuales poseen gran relevancia ecológica y económica (Ramírez \& Cáceres 1991). Esta subfamilia ha tenido posición controversial, desde ser considerada un subgénero (Beaquert 1948), tomar la posición de familia (Leme 1973), hasta actualmente ser considerada una subfamilia (Bouchet \& Rocroi 2005). La mayoría de estas especies se encuentran descritas a partir de su concha. Esto ha generado numerosas controversias sobre la determinación correcta de algunas especies ya que los caracteres conquiliológicos resultan ser muy plásticos (Borda \& Ramírez 2013). 
Para la vertiente oriental del sur de los Andes peruanos se han reportado cuatro especies de Megalobulimus (Miller): $M$. popelairianus (Nyst, 1845), M. maximus (Sowerby, 1825), $M$. leucostoma (Sowerby, 1835) y M. florezi Borda \& Ramírez, 2013. Megalobulimus leucostoma presenta la particularidad de tener tres subespecies descritas, dos de las cuales han sido reportadas para el departamento del Cusco, M. leucostoma leucostoma (Sowerby, 1835) y M. leucostoma weyrauchi (Bequaert, 1949). La tercera subespecie, $M$. leucostoma lacunosus (d'Orbigny, 1835), ha sido descrita para el departamento de Puno y en Bolivia. En las descripciones originales de estas subespecies la localidad tipo de $M$. l weyrauchi no es totalmente clara, además no aclaran las diferencias entre las subespecies $M$. l. leucostoma y M. l. lacunosus. La imprecisión de las descripciones originales lleva a considerar incierto el estatus taxonómico de $M$. leucostoma, pues existen características conquiliológicas entre las tres subespecies que ponen en duda su unidad como especie. El objetivo del presente trabajo es evaluar, a nivel morfométrico, similitudes y diferencias de las conchas entre las tres subespecies contrastadas con sus distribuciones geográficas las cuales podrían ayudar a una mejor caracterización y definición del estatus taxonómico de estos taxa.

\section{Material y métodos}

Las colectas se llevaron a cabo durante febrero, marzo y junio del 2012 en las provincias de La Convención, Urubamba y Calca, pertenecientes al departamento de Cusco, y la provincia de Carabaya en el departamento de Puno. El método de colecta fue búsqueda directa. Los especímenes colectados fueron depositados en la colección malacológica del Departamento de Malacología y Carcinología del Museo de Historia Natural de la Universidad Nacional Mayor de San Marcos (MUSM). Asimismo, se consideraron ejemplares tipo custodiados en el Natural History Museum of the United Kingdom (NHM-UK), correspondientes a Helix mathiusii (d'Orbigny, 1835), dos de Helix lacunosus (d'Orbigny, 1835); el primero es considerado sinónimo de $M$. l. leucostoma. Además, se consideró material tipo de $M$. leucostoma weyrauchi custodiado en el Museum of Comparative Zoology of Harvard University (MCZ) y Academy of Natural Sciences of Drexel University (ANSP) (Tabla 1 y Fig. 1).

Con la finalidad de realizar la discriminación de las subespecies de Megalobulimus leucostoma se realizó un análisis morfométrico de las conchas, tanto del material colectado como de los custodiados en colecciones. Para esto se realizaron dos evaluaciones en morfometría, la clásica y la geométrica. Fueron evaluados 83 individuos adultos, considerados así por el engrosamiento del peristoma, 71 de los cuales corresponden a $M$. leucostoma leucostoma, tres a $M$. leucostoma lacunosus, cinco a $M$. leucostoma weyrauchi, dos a Helix mathiusii y dos a Helix lacunosus.

Para la morfometría clásica, se emplearon seis variables continuas las que fueron obtenidas a través de imágenes digitales de la concha de los especímenes en el programa IMAGEJ (versión 1.63, http://rsb.info.nih.gov/nih-image). Las variables consideradas fueron: Altura de la concha (AC), diámetro mayor de la concha (DM), largo de la abertura (LB), ancho de la abertura (AB), espira (ESP) y posición del diámetro mayor (PDM) (Figura 2). Las seis variables fueron sometidas a análisis de componentes principales (ACP) mediante el programa PAST ver 3.0 (Hammer et al. 2001). Los datos fueron transformados a logaritmos previamente.

Para la morfometría geométrica se consideraron 12 landmarks, los cuales fueron digitalizados con el programa TpsDig ver. 2.16 (Rohlf 2010) (Fig. 3). Estos fueron considerados en base al trabajo de Schilthuizen y Haase (2010). Las coordenadas fueron ingresadas al programa MorphoJ 1.05 (Klingenberg 2011), realizándose antes un alineamiento de las coordenadas iniciales usando el método generalizado ortogonal de Procrustes (Rohlf \& Slice 1990), se generó una matriz de covarianza, la que permitió realizar el análisis de componentes principales. Debido al número desbalanceado de individuos de cada subespecie, se consideraron las tres primeras componentes principales para realizar un

Tabla 1. Procedencia de los especímenes empleados en el presente estudio.

\begin{tabular}{|c|c|c|c|c|}
\hline Subespecie & $\begin{array}{l}\text { Museo de } \\
\text { custodia }\end{array}$ & Procedencia & Coordenadas & $\begin{array}{c}\text { \# de } \\
\text { ejemplares }\end{array}$ \\
\hline \multirow{11}{*}{$\begin{array}{c}\text { Megalobulimus leucostoma } \\
\text { leucostoma }\end{array}$} & \multirow{11}{*}{ MUSM } & Perú, Cusco, La Convención, Pavayoc & $12^{\circ} 51^{\prime} 39.52^{\prime \prime} \mathrm{S}-2^{\circ} 41^{\prime} 13.9^{\prime \prime} \mathrm{W}$ & 13 \\
\hline & & Perú, Cusco, La Convención, Huyro & $12^{\circ} 58^{\prime} 39,5^{\prime \prime} \mathrm{S}-72^{\circ} 33^{\prime} 21,9^{\prime \prime} \mathrm{W}$ & 4 \\
\hline & & Perú, Cusco, La Convención, Santa María & $13^{\circ} 00^{\prime} 16.2^{\prime \prime} \mathrm{S}-72^{\circ} 37^{\prime} 57.1^{\prime \prime} \mathrm{W}$ & 8 \\
\hline & & Perú, Cusco, La Convención, Chaullay & $13^{\circ} 00^{\prime} 34.6^{\prime \prime} \mathrm{S}-72^{\circ} 38^{\prime} 34.9^{\prime \prime} \mathrm{W}$ & 2 \\
\hline & & Perú, Cusco, La Convención, Sambaray & $12^{\circ} 51^{\prime} 49.50^{\prime \prime} \mathrm{S}-72^{\circ} 42^{\prime} 16.2^{\prime \prime} \mathrm{W}$ & 18 \\
\hline & & Perú, Cusco, La Convención, Echarate & $12^{\circ} 46^{\circ} 51.0^{\prime \prime} \mathrm{S}-72^{\circ} 33^{\prime} 59.7^{\prime \prime} \mathrm{W}$ & 10 \\
\hline & & Perú, Cusco, La Convención, Potrero & $12^{\circ} 53^{\prime} 41.0^{\prime \prime} \mathrm{S}-72^{\circ} 43^{\prime} 42.6^{\prime \prime} \mathrm{W}$ & 4 \\
\hline & & Perú, Cusco, La Convención, Uchumayo & $12^{\circ} 57^{\prime} 26.0^{\prime \prime} \mathrm{S}-72^{\circ} 40^{\prime} 25.8^{\prime \prime} \mathrm{W}$ & 1 \\
\hline & & Perú, Cusco, La Convención, Huayanay & $12^{\circ} 48^{\prime} 40.3^{\prime \prime} \mathrm{S}-72^{\circ} 38^{\prime} 42.6^{\prime \prime} \mathrm{W}$ & 6 \\
\hline & & Perú, Cusco, La Convención, Santa Teresa & $13^{\circ} 06^{\prime} 17^{\prime \prime} \mathrm{S}-72^{\circ} 37^{\prime} 51^{\prime \prime} \mathrm{W}$ & 4 \\
\hline & & Perú, Cusco, Calca, Lares & $13^{\circ} 05^{\prime} \mathrm{S}-72^{\circ} 13^{\prime} \mathrm{W}$ & 2 \\
\hline \multirow{2}{*}{$\begin{array}{c}\text { Megalobulimus leucostoma } \\
\text { lacunosus }\end{array}$} & \multirow{2}{*}{ MUSM } & Perú, Puno, Chacaneque & $13^{\circ} 18^{\prime} 53^{\prime \prime} \mathrm{S}-70^{\circ} 17^{\prime} 08^{\prime \prime} \mathrm{W}$ & 2 \\
\hline & & Perú, Madre de Dios, Tambopata & $14^{\circ} 14^{\prime} \mathrm{S}-89^{\circ} 11^{\prime} \mathrm{W}$ & 1 \\
\hline \multirow{2}{*}{$\begin{array}{c}\text { Megalobulimus leucostoma } \\
\text { weyrauchi }\end{array}$} & ANSP & Peru, Cusco & - & 1 \\
\hline & $\mathrm{MCZ}$ & Perú, Cusco & - & 4 \\
\hline Helix mathiusii & NHM-UK & Perú, Cusco & - & 2 \\
\hline Helix lacunosus & NHM-UK & Bolivia, Cochabamba, Tutulima & - & 2 \\
\hline
\end{tabular}




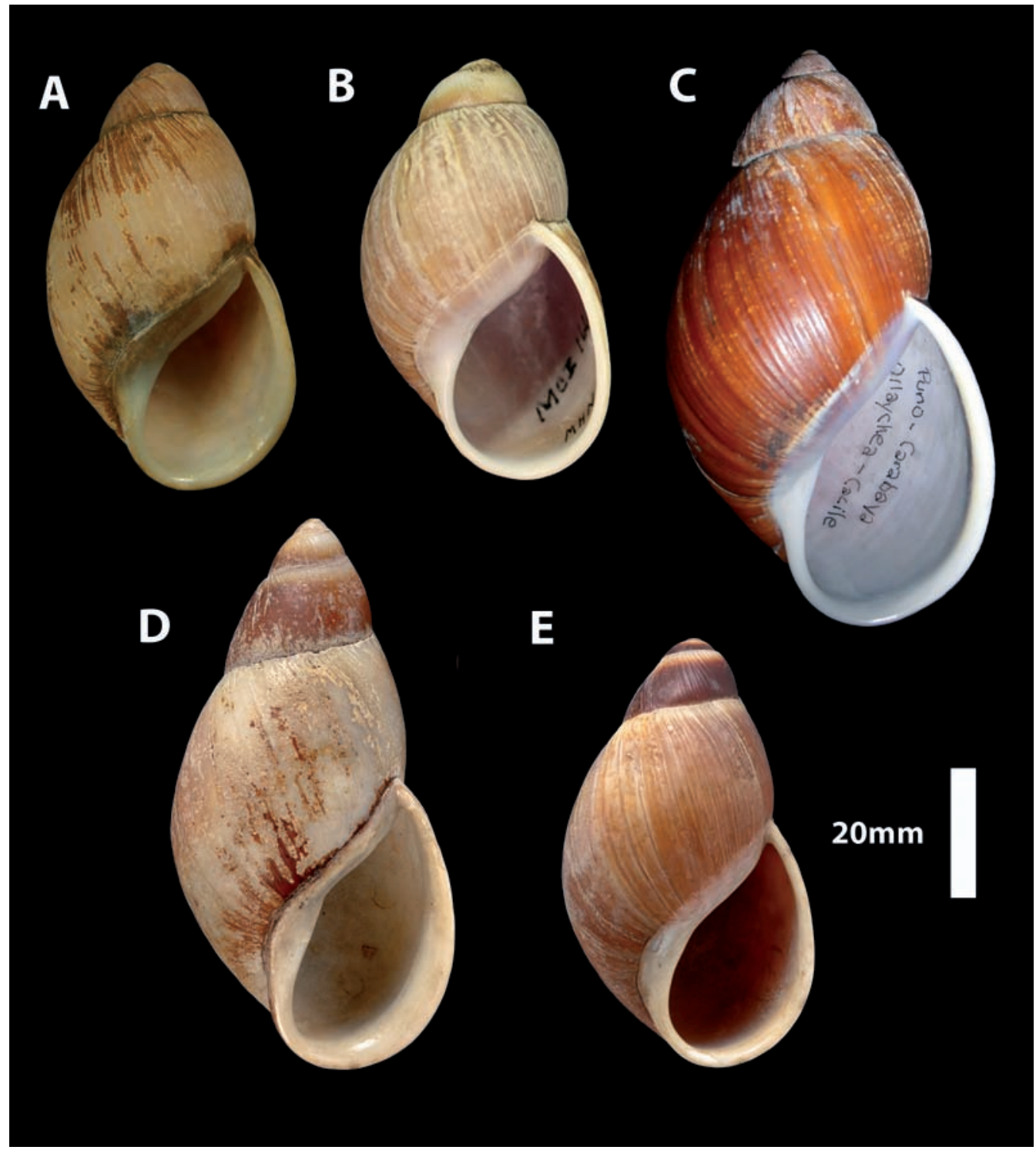

Figura 1. Subespecies de Megalobulimus leucostoma en vista frontal.

(A) M. leucostoma procedente de Pavayoc, Cusco.

(B) Holotipo de M. leucostoma weyrauchi, MCZ 166295. Cortesía del MCZ.

(C) M. leucostoma lacunosus procedente de Carabaya, Puno.

(D) Helix lacunosus, sinonimia para M. leucostoma lacunosus, sintipo procedente de la localidad de Tutulima, Cochabamba, Bolivia, $\mathrm{NH}$ MUK 1854.12.4.213. Cortesía del NHMUK.

(E) Helix mathiusii, sinonimia para M. I. leucostoma, sintipo procedente de Perú NHMUK 1854.12.4.214. Cortesía del NHMUK.
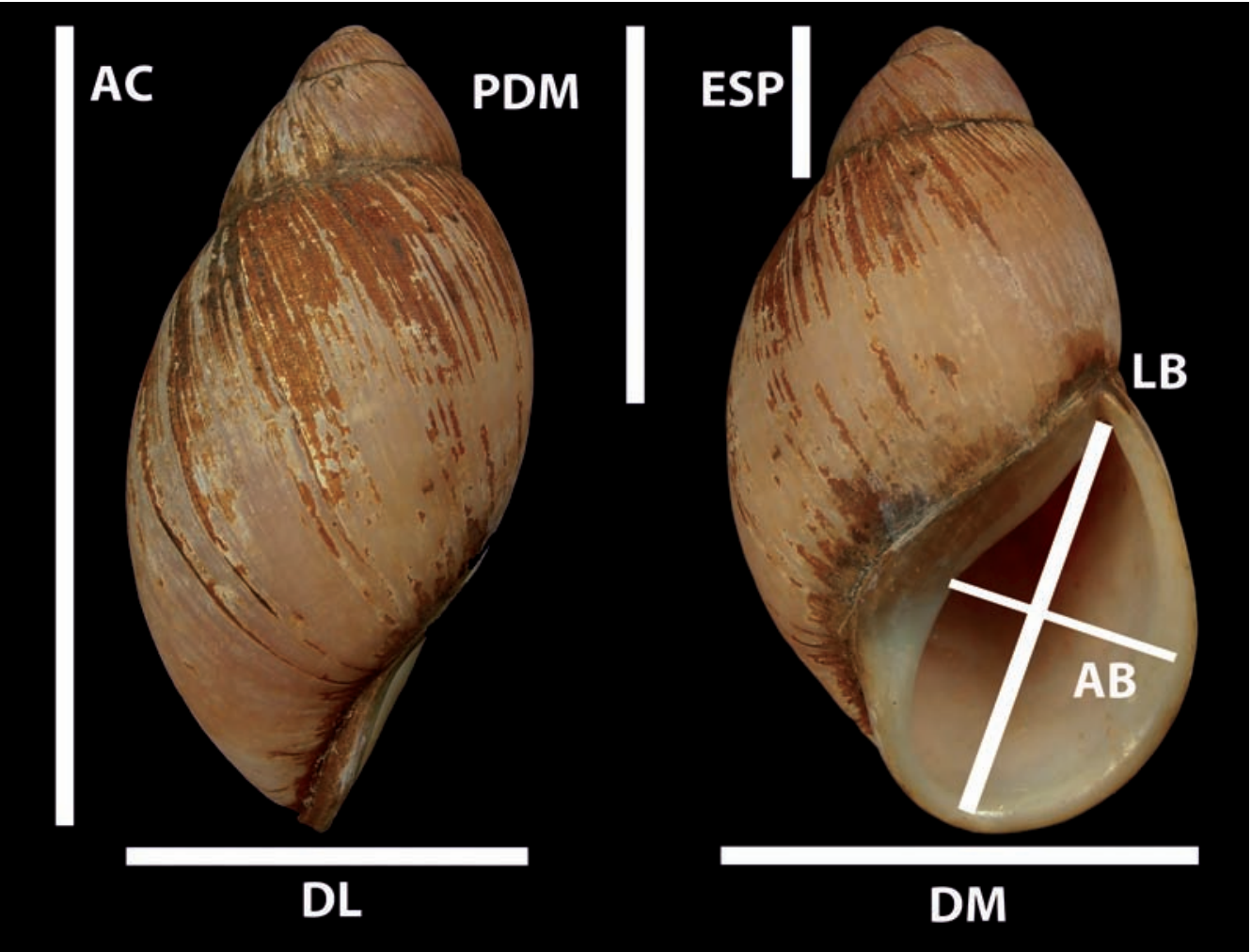

Figura 2. Variables de la concha consideradas para el análisis morfométrico clásico.

AC: Altura de la concha.

DM: Diámetro mayor de la concha.

LB: Largo de la abertura. $A B$ : Ancho de la abertura. ESP: Espira.

PDM: Posición del diámetro mayor. 
Tabla 2. Estadísticos básicos para las seis medidas obtenidas a partir de la concha de las subespecies de Megalobulimus leucostoma.

\begin{tabular}{|c|c|c|c|c|c|}
\hline Medidas & M. l. leucostoma $(\mathrm{n}=71)$ & M. l. lacunosus $(\mathrm{n}=3)$ & M. l. weyrauchi $(\mathrm{n}=5)$ & Helix mathiusii $(\mathrm{n}=2)$ & Helix lacunosus $(\mathrm{n}=2)$ \\
\hline $\begin{array}{c}\text { Altura de la } \\
\text { concha }\end{array}$ & $70.53 \pm 3.66$ & $87.38 \pm 1.88$ & $62.1 \pm 3.4$ & $64.16 \pm 1.17$ & $85.31 \pm 0.87$ \\
\hline $\begin{array}{l}\text { Diámetro mayor } \\
\text { de la concha }\end{array}$ & $39.31 \pm 2.4$ & $47.82 \pm 2.33$ & $36.96 \pm 1.03$ & $39 \pm 0.36$ & $43.53 \pm 0.93$ \\
\hline $\begin{array}{c}\text { Largo de la } \\
\text { abertura }\end{array}$ & $34.36 \pm 2.45$ & $43.67 \pm 0.98$ & $37.75 \pm 2.75$ & $32.60 \pm 0.36$ & $40.08 \pm 0.08$ \\
\hline $\begin{array}{c}\text { Ancho de la } \\
\text { abertura }\end{array}$ & $18.5 \pm 1.27$ & $22.82 \pm 1.64$ & $20 \pm 1$ & $18.28 \pm 0.33$ & $21.5 \pm 0.22$ \\
\hline Espira & $16.99 \pm 1.44$ & $23.58 \pm 0.99$ & $14.56 \pm 0.66$ & $13.15 \pm 0.3$ & $23.51 \pm 0.69$ \\
\hline $\begin{array}{c}\text { Posición del } \\
\text { diámetro mayor }\end{array}$ & $37 \pm 2.23$ & $34.36 \pm 1.31$ & $37.78 \pm 2.5$ & $34.36 \pm 1.31$ & $47.22 \pm 0.89$ \\
\hline
\end{tabular}

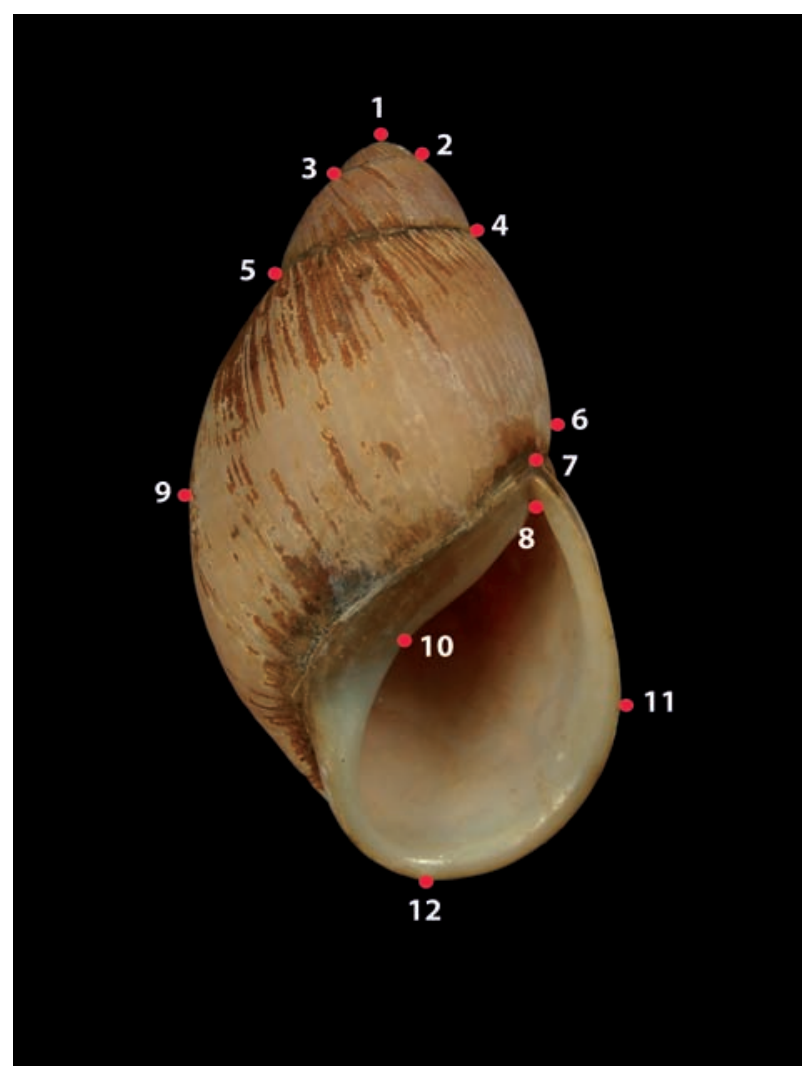

Figura 3. Landmarks utilizados en el estudio basados en Schilthuizen \& Haase (2010).

MANOVA no paramétrico mediante el programa PAST ver 3.0. Este MANOVA se realizó con el objetivo de comparar las medias multivariadas para las tres subespecies considerando el índice de distancia Euclidiana y de Mahalanobis. Finalmente se realizó un análisis discriminante para mejorar la distinción entre los grupos evaluados a partir de las tres primeras componentes principales donde ya se vería reflejada la variación original de los landmarks. Para estos análisis se consideraron tres grupos basados en su descripción como subespecies, estos fueron: grupo $1(M$. l. leucostoma + Helix mathiusii); grupo 2 (M. l. lacunosus + Helix lacunosus) y grupo 3 (M. l. weyrauchi).

Los datos de coordenadas geográficas procedentes de las colectas y del material de colección fueron considerados para modelar un mapa de distribución en el programa Arc Map 10. Se consideró la clasificación de zonas de vida de Holdridge (1947) para caracterizar el hábitat de las especies colectadas.

\section{Resultados}

Morfometría clásica.- Se calcularon los estadísticos básicos (media y desviación estándar) para las seis medidas propuestas (Tabla 2). Luego éstas fueron normalizadas y sometidas al Análisis de Componentes Principales (ACP). Las dos primeras componentes explicaron un $92.964 \%$ de la varianza total. La primera componente acumuló un $71.169 \%$ de la varianza total y la variable que explicó mejor dicha varianza fue la longitud de la espira de la concha. La segunda componente acumuló $21.795 \%$ de la variabilidad total. La variable que mejor explicó la variación en esta componente también fue la longitud de la espira seguida del ancho de la apertura (Tabla 3).

En la gráfica de dispersión de puntos de las primeras dos componentes principales (Fig. 4), se observa la gran variabilidad de los individuos de $M$. leucostoma, dentro de este morfoespacio se ubican los ejemplares tipo de Helix mathiusii y de M. leucostoma weyrauchi. Mientras que, por fuera de este morfoespacio, se sitúan $M$. leucostoma lacunosus (Puno) y los ejemplares tipo de Helix lacunosus como las conchas de mayores proporciones.

Se observó una gran variación en el tamaño de M. leucostoma leucostoma, donde los ejemplares tipo de M. leucostoma weyrauchi y de $H$. mathiusii se ubican junto con las de menor tamaño.

Morfometría geométrica.- A partir del análisis de componentes principales para 24 coordenadas de los 12 landmarks se obtuvieron 20 componentes principales (PC). Las primeras dos componentes explicaron el $48.748 \%$ de la varianza total, la primera con $35.862 \%$ y la segunda con $12.88 \%$ (Fig. 5). En la componente principal 1, la forma varió desde conchas más anchas, de protoconcha corta y de apertura más grande en su

Tabla 3. Matriz de estructura del análisis de componentes principales para 83 conchas de las especies en estudio.

\begin{tabular}{lccc}
\hline \multirow{2}{*}{ Variables } & \multicolumn{3}{c}{ Componentes principales } \\
& $\mathbf{1}$ & $\mathbf{2}$ & $\mathbf{3}$ \\
\hline Altura de la concha & 0.39254 & 0.001773 & 0.40445 \\
Diámetro de la concha & 0.29732 & 0.33467 & 0.70615 \\
Espira & 0.59436 & -0.69691 & -0.15362 \\
Posición del diámetro mayor & 0.412 & 0.024925 & -0.0189 \\
Altura de la apertura & 0.36456 & 0.41241 & -0.45981 \\
Ancho de la apertura & 0.31874 & 0.48126 & -0.31999 \\
Total de varianza explicada & $71.169 \%$ & $21.795 \%$ & $2.69 \%$ \\
\hline
\end{tabular}




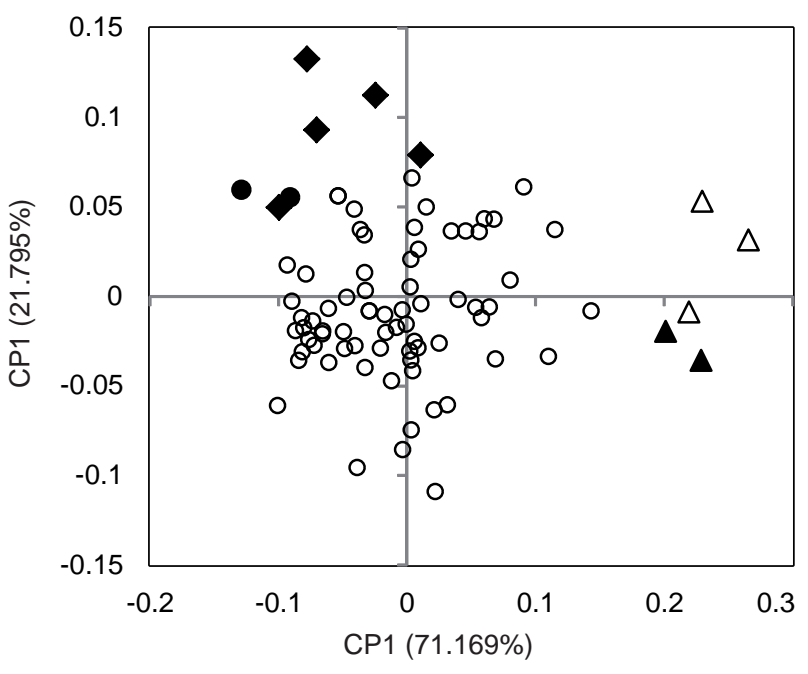

O M. I. leucostoma

$\Delta$ Helix lacunosus

M. I. weyrauchi

Helix mathiusii

$\triangle M$. I. lacunosus

Figura 4. Análisis de componentes principales basado seis medidas obtenidas a partir de 83 conchillas para la discriminación entre las tres subespecies de Megalobulimus leucostoma y ejemplares tipo. El porcentaje de variación para cada componente se indica entre paréntesis.

extremo más negativo a conchas más delgadas, con protoconcha más pronunciada y de apertura más pequeñas en su porción positiva. Los landmarks que acumularon mayor varianza para esta componente estuvieron relacionados con la espira (landmarks 1 y 2) (Tabla 4). En el caso de la componente principal 2, se observan conchas con espira corta en su extremo más negativo, cuya forma varió a conchas con espira más pronunciada en el extremo más positivo. $\mathrm{Al}$ igual que la componente principal 1 , los landmarks que acumularon mayor varianza para esta componente fueron los landmarks 1 y 2 (Tabla 4).

En el gráfico se observa que $M$. l. leucostoma presenta diversas formas que abarcan desde formas delgadas con espira aguda hasta formas semiovaladas con espiras obtusas. Los individuos de $M$. l. lacunosus se ubican preferentemente dentro de los cuadrantes correspondientes a las conchas con forma delgada y espira más pronunciada, siendo los individuos tipo de $H$. lacunosus aquellos que se diferenciaron mejor por su forma más delgada. Por otro lado, los individuos de $M$. l. weyrauchi y $H$. mathiusii se diferenciaron como los ejemplares con ápex más corto y vuelta corporal más expandida sin mostrar mucha diferenciación del morfoespacio de M. leucostoma leucostoma (Fig. 5).

A partir de los valores para los tres primeros componentes principales se desarrolló un análisis PERMANOVA o MANOVA no paramétrico. Para esto se consideraron tres grupos basados en cada subespecie de $M$. leucostoma: grupo 1 ( $M$. l. leucostoma + Helix mathiusii); grupo 2 (M. l. lacunosus + Helix lacunosus) y grupo 3 (M. l. weyrauchi). PERMANOVA no reveló diferencias significativas entre los tres grupos (distancia Euclidiana: $F$ =5.728, $\mathrm{P}<0.001$; distancia de Mahalanobis: $F=4.951, \mathrm{P}<$ $0.001)$. Sin embargo, si se examinan los valores considerando pares de grupos es posible observar, en el caso de la distancia Euclidiana, una clara similitud entre las subespecies leucostoma y weyrauchi. Mientras que en el caso de los pares $M$. l. lacunosus

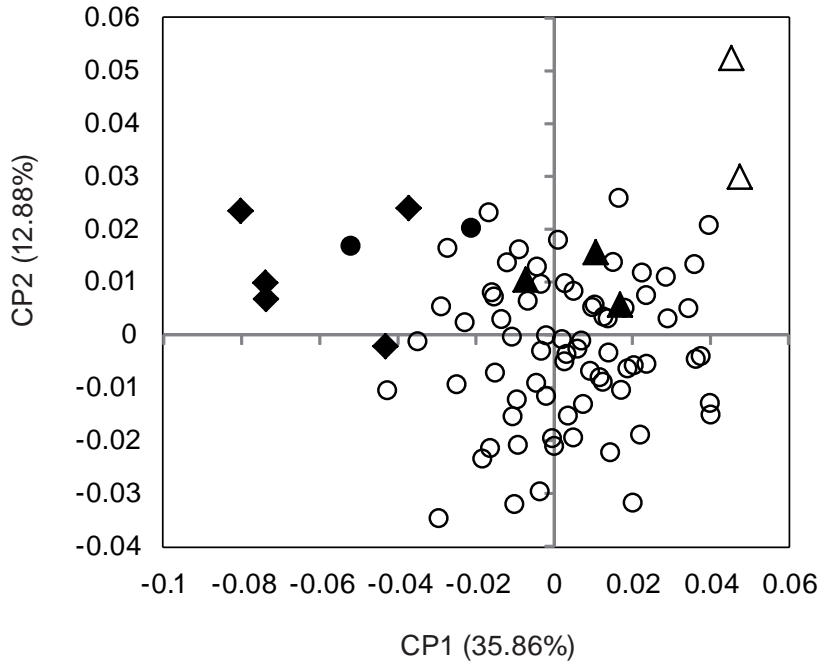

\section{OM. I. leucostoma $\Delta$ Helix lacunosus M. I. weyrauchi}

Helix mathiusii

A. M. lacunosus

Figura 5. Análisis de componentes principales basado en variables de forma para la discriminación entre las tres subespecies de Megalobulimus leucostoma y ejemplares tipo. El porcentaje de variación para cada componente se indica entre paréntesis.

Tabla 4. Matriz de estructura de las cuatro primeras componentes principales para las coordenadas de los 12 landmarks utilizados.

\begin{tabular}{|c|c|c|c|c|}
\hline & PC1 & PC2 & PC3 & PC4 \\
\hline $\mathrm{x} 1$ & -0.012821 & 0.319020 & -0.096432 & 0.003716 \\
\hline y1 & 0.498506 & 0.210085 & 0.042753 & 0.164806 \\
\hline$x 2$ & 0.079255 & 0.332015 & -0.035472 & 0.089456 \\
\hline y2 & 0.291036 & -0.078494 & 0.005197 & -0.002814 \\
\hline$x 3$ & -0.184434 & 0.080919 & -0.184978 & -0.161165 \\
\hline y3 & 0.071813 & 0.028658 & -0.135326 & -0.060551 \\
\hline$x 4$ & 0.081609 & 0.129819 & 0.042635 & 0.067133 \\
\hline $\mathrm{y} 4$ & -0.022849 & -0.284958 & -0.052685 & -0.093376 \\
\hline $\mathrm{x} 5$ & -0.069673 & -0.219091 & -0.051349 & -0.088025 \\
\hline y5 & -0.220655 & -0.206762 & -0.106868 & -0.201052 \\
\hline$x 6$ & -0.031914 & -0.199290 & -0.000729 & 0.134719 \\
\hline y6 & -0.292434 & 0.204729 & 0.157102 & 0.473087 \\
\hline$x 7$ & -0.114788 & -0.263246 & -0.058426 & 0.151766 \\
\hline y7 & -0.338916 & 0.042351 & -0.057242 & 0.126941 \\
\hline$x 8$ & -0.014239 & -0.256633 & -0.308946 & 0.116012 \\
\hline y8 & -0.287791 & 0.058757 & -0.166630 & -0.098571 \\
\hline$\times 9$ & 0.251628 & -0.298582 & 0.327527 & 0.105137 \\
\hline y9 & -0.154369 & -0.102215 & 0.694639 & -0.125136 \\
\hline x10 & 0.160123 & 0.013503 & 0.256303 & -0.250565 \\
\hline y10 & -0.064606 & 0.215789 & 0.058906 & -0.121606 \\
\hline x11 & -0.070387 & 0.076755 & 0.053466 & -0.411112 \\
\hline y11 & 0.265691 & 0.158838 & -0.226640 & -0.373852 \\
\hline$x 12$ & -0.074360 & 0.284813 & 0.056402 & 0.242926 \\
\hline y12 & 0.254573 & -0.246777 & -0.213207 & 0.312123 \\
\hline
\end{tabular}




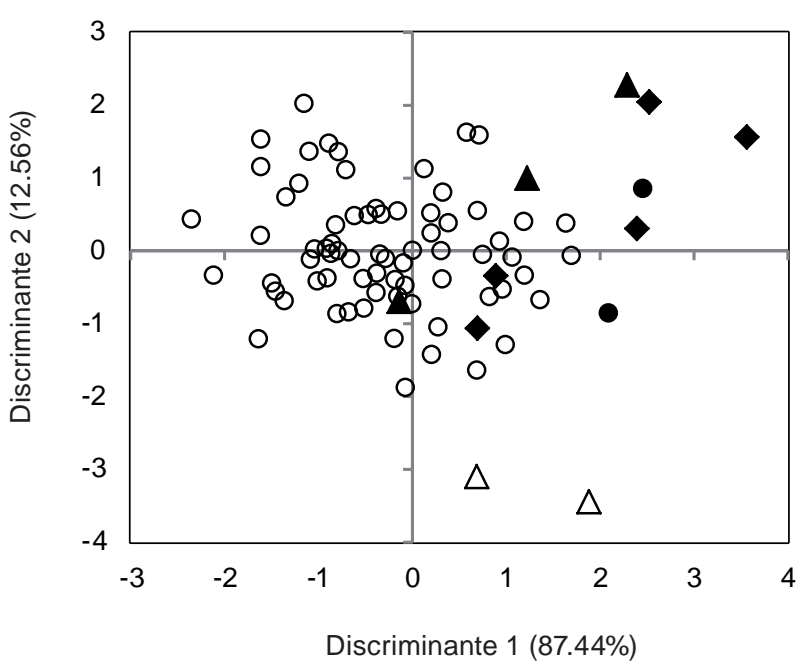

OM.l. leucostoma $\Delta$ Helix lacunosus $\wedge$ M. I. weyrauchi

Helix mathiusi

$\Delta$ M. I. lacunosus

Figura 6. Análisis discriminante basado en las tres primeras componentes principales obtenidas a partir de variables de forma para la discriminación entre las tres subespecies de Megalobulimus leucostoma y ejemplares tipo. El porcentaje de variación para cada factor discriminante se indica entre paréntesis.

- M. l. leucostoma y M. l. lacunosus - M. l. weyrauchi la similitud es no significativa. Para el caso de la distancia de Mahalanobis, es posible observar que existe una similitud significativa entre los pares $M$. l. lacunosus - M. l. leucostoma y $M$. l. leucostoma M. l. weyrauchi (Tabla 5). Por último, el análisis discriminante demuestra que aún es posible notar que aunque existe superposición entre los morfoespacios de los grupos, es la componente principal 2, la que contribuye con una ligera diferenciación entre los grupos 2 y 3 del grupo 1 (M. l. leucostoma + Helix mathiusii) (Fig. 6).

Distribución geográfica.- Los datos de distribución geográfica producto de las colectas y de la colecciones científicas resaltan el carácter endémico para la subespecie $M$. l. leucostoma, la cual se ve restringida a las provincias de La Convención y Calca, en el departamento de Cusco (Fig. 7). El área donde fue colectada esta subespecie corresponde a la zona de vida "Bosque Húmedo tropical” (bh-t) según el sistema de Holdrigde (Holdridge 1947). En la provincia de La Convención, las localidades más distantes fueron Huyro y Echarate las cuales están separadas por 70 kilómetros. Todas las localidades se encuentran próximas al Río Vilcanota (Urubamba), excepto la localidad de Santa Teresa. El punto de colecta a mayor altitud fue en Huyro, la cual también corresponde a la localidad más al sur dentro de la provincia de La Convención. En el caso de $M$. l. lacunosus, fue colectada únicamente en la localidad de Chacaneque en Puno, la cual corresponde a la zona de vida "Bosque húmero tropical" (bh-t). Para M. l. weyrauchi, solo se menciona "Cusco" tanto en la descripción original como en las etiquetas de los ejemplares tipo custodiados en la colección del MCZ.

\section{Discusión}

Inicialmente las subespecies del complejo $M$. leucostoma habían sido descritas como especies independientes, Bulimus leucostoma Sowerby, 1835 y Helix lacunosus d'Orbigny, 1835. La diagnosis de estas dos especies se basó en características de la concha tales como forma, tamaño de la espira, características de la superficie, vueltas nepiónicas, entre otras. Además que fueron descritas para Perú (Cusco) y Bolivia (Cochabamba) respectivamente. Bequaert (1948) fue quién en su revisión de la Familia Strophocheilidae consideró a H. lacunosus como una subespecie de Strophocheilus (Megalobulimus) leucostoma. Al año siguiente, Bequaert (1949) describió a Strophocheilus (Megalobulimus) leucostoma weyrauchi a partir de 9 individuos (el holotipo y 8 paratipos). En comparación con el típico Strophocheilus leucostoma (ahora Megalobulimus leucostoma), Bequaert describió esta subespecie como más pequeña y proporcionalmente más ancha a nivel de la vuelta corporal. El presente estudio abarcó localidades citadas en la literatura para $M$. leucostoma y nuevas localidades en el Perú, además de incluir especímenes tipo para una mejor caracterización de las subespecies que componen a M. leucostoma.

Los métodos de morfometría tradicional referidos a medidas lineales básicas (como ancho y altura) son ampliamente usados para comparar la forma de diferentes organismos, sin embargo, esta información resulta limitada (Smith \& Hendricks 2013). Uno de los principales problemas dentro del género Megalobulimus es la constancia y homogeneidad en sus

Tabla 5. P-values de pares de grupos para las tres subespecies producto del MANOVA no paramétrico a partir de los valores de las tres primeras componentes principales basadas en variables de forma. Se considera valores de distancia Euclidiana y de Mahalanobis. El asterisco $\left(^{*}\right)$ indica los valores significativos.

\begin{tabular}{|c|c|c|c|}
\hline Distancia Euclidiana & & & \\
\hline Subespecies & M. l. leucostoma & M. l. lacunosus & M. l. weyrauchi \\
\hline M. l. leucostoma & & 0.0673 & $0.0003^{*}$ \\
\hline M. l. lacunosus & 0.0673 & & 0.2692 \\
\hline M. l. weyrauchi & $0.0003^{*}$ & 0.2692 & \\
\hline \multicolumn{4}{|c|}{ Distancia de Mahalanobis } \\
\hline Subespecies & M. l. leucostoma & M. l. lacunosus & M. l. weyrauchi \\
\hline M. l. leucostoma & & $0.0167^{*}$ & $0.0002^{*}$ \\
\hline M. l. lacunosus & $0.0167^{*}$ & & 0.7667 \\
\hline M. l. weyrauchi & $0.0002^{*}$ & 0.7667 & \\
\hline
\end{tabular}




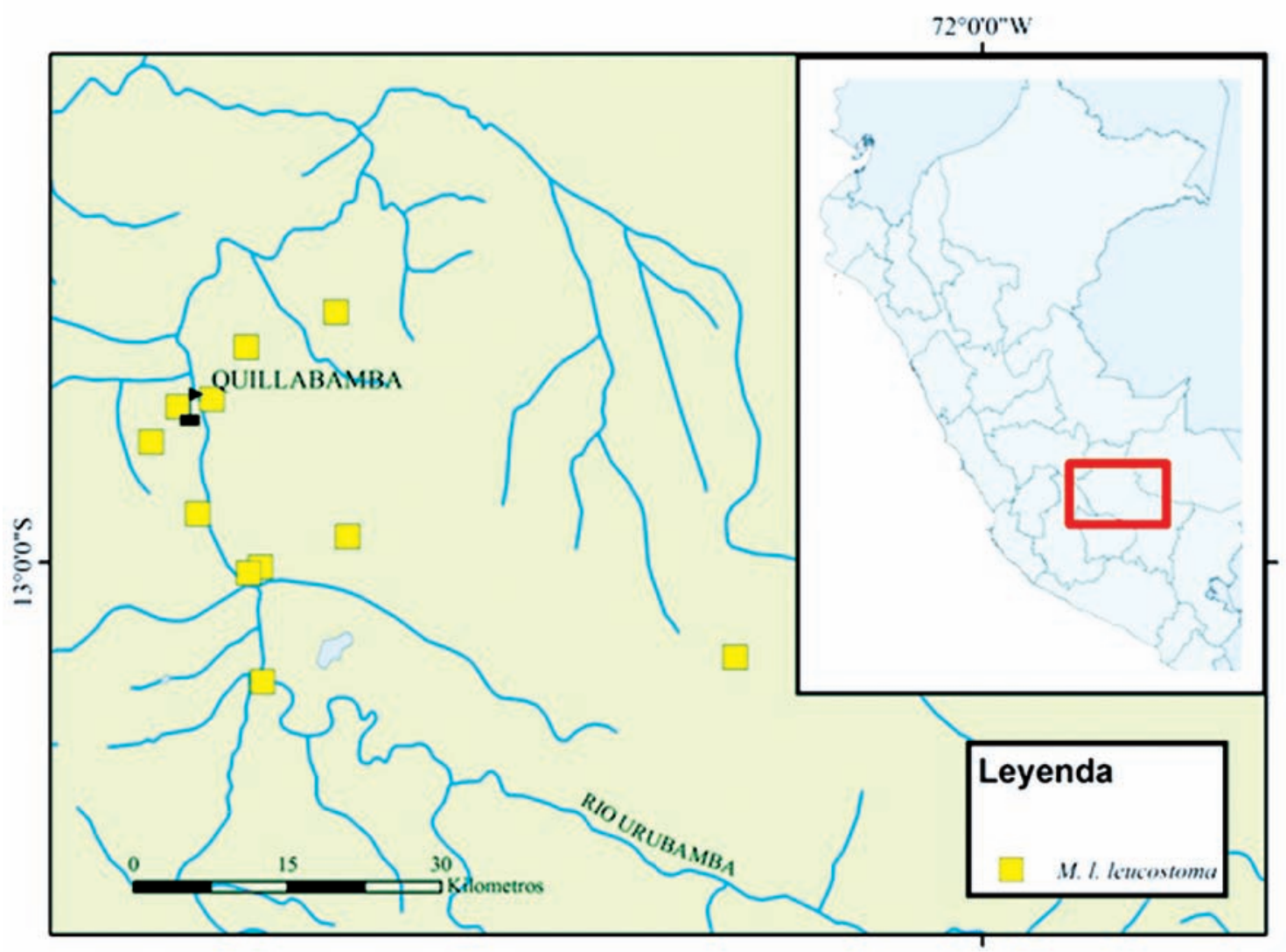

Figura 7. Mapa de procedencia del material evaluado (Suroriente del Perú) para M. leucostoma leucostoma en el departamento de Cusco. Los ríos están diferenciados como líneas en azul.

caracteres conquiliológicos y anatómicos (Leme 1973). El caso del complejo $M$. leucostoma es un claro ejemplo en el cual la variabilidad intraespecífica es alta, al menos al referirse a los caracteres conquiliológicos. Por este motivo se abordaron dos diferentes metodologías en morfometría para diferenciar entre las subespecies de este complejo.

Los resultados obtenidos a partir de ambos análisis de morfometría proveerían escenarios contradictorios. Sin embargo, es necesario interpretarlos de manera integrada. El análisis multivariado de las medidas lineales nos permitió discriminar en base a tamaño y forma, siendo diferenciadas las subespecies $M$. l. leucostoma y $M$. l. lacunosus en base a sus diferentes tamaños y características de la espira. Además, se destaca el amplio rango de variación que presenta $M$. l. leucostoma y por esta razón no se consiguió una discriminación significativa de $M$. l. weyrauchi. En el caso de la morfometría geométrica, la cual considera la forma, también demostró que M. l. leucostoma no solo presenta amplia variabilidad en los tamaños sino también en las formas. Este morfoespacio de forma incluyó a $M$. l. lacunosus, ya que algunas conchas a pesar de tener un tamaño menor al promedio de $M$. l. lacunosus, presentan una forma similar. Al igual que en la morfometría clásica, no se encontró una diferenciación significativa de M. l. weyrauchi. Ambos análisis demuestran que esta subespecie pertenece al gran rango de variabilidad de $M$. $l$. leucostoma tanto por tamaño como por forma. Mientras que $M$. l. lacunosus aun presentando una forma parecida, la diferencia de tamaño con $M$. l. leucostoma es marcada. Asimismo, en otros estudios morfológicos y moleculares que estamos desarrollando se reafirma la distancia entre $M$. l. lacunosus y $M$. l. leucostoma
En el caso de M. l. weyrauchi, fue descrita para Cusco sin más especificaciones por Bequaert (1949), no proporcionó datos exactos de su distribución. Las únicas localidades de Cusco donde se han reportado especímenes de Megalobulimus corresponden a las provincias de La Convención y Calca (Flórez 1970), ambas con diferentes zonas de vida. Además, las características conquiológicas de la descripción no son suficientes para justificar su estatus como subespecie. Los análisis morfométricos clásico y geométrico lo incluyen o lo acercan, respectivamente, al morfoespacio de $M$. l. leucostoma. Además, el MANOVA y el análisis discriminante no proveen un sustento suficiente como para distinguir a $M$. l. weyrauchi como una subespecie, sino más bien que constituye parte de la variabilidad intrapoblacional de M. leucostoma leucostoma.

La diversidad de formas y tamaños observados para $M$. $l$. leucostoma no se encuentran separadas geográficamente. Por el contrario, en una misma población es posible observar no sólo diferentes tamańos y formas sino también ejemplares levógiros (Blume 1920).

A partir de los resultados, M. leucostoma lacunosus debería recuperar su estatus de especie, quedando como Megalobulimus lacunosus (d'Orbigny, 1835), Por el contrario, M. l. weyrauchi (Bequaert, 1949) constituiría una variación dentro de la gran gama de formas y tamaños de $M$. leucostoma, constituyéndose en un sinónimo de ésta.

\section{Agradecimientos}

Agradecemos a André Ampuero por su colaboración en el análisis morfométrico y a John Ablet (NHMUK) y Adam Baldinger (MCZ) por proveer imágenes digitales de los ejemplares tipo. 


\section{Literatura citada}

Bequaert J.C. 1948. Monograph of the Strophocheilidae, a Neotropical family of terrestrial mollusks. Bulletin of the Museum of Comparative Zoology 100:1-210.

Bequaert J. C. 1949. Three new south american land snails (Strophocheilus, Thaumastus, Drymaeus). The Nautilus 62:113 $-115$.

Blume W. 1920. Vier neue Landschnecken. Archiv für Molluskenkunde 52:127-130.

Borda V. \& R. Ramírez. 2013. Re-characterization of the Red-lip Megalobulimus (Gastropoda: Strophocheilidae) from Peru with description of a new species. Zoologia $(\mathrm{Cu}-$ ritiba) 30: 675-691. http://dx.doi.org/10.1590/S198446702013005000008 .

Bouchet P. \& J.-P. Rocroi. 2005. Classification and nomenclator of gastropod families. Malacologia 47:397pp.

Flórez A. 1970. Moluscos de interés económico del Oriente Cusqueño (Familia Acavidae). Publicaciones del Departamento Académico de Zoología y Entomología - Universidad Nacional de San Antonio Abad del Cusco 1:27-31.

Hammer Ø.; D. Harper \& P. Ryan. 2001. Past: Paleontological Statistics Software Package for education and data analysis. . Paleontologia Electronica 4:1-9.

Holdridge L. R. 1947. Determination of World Plant Formations from Simple Climatic Data. Science 105:367-368. doi: 10.1126/ science.105.2727.367
Klingenberg C.P. 2011. MorphoJ: an integrated software package for geometric morphometrics. Molecular Ecology Resources 11:353-357. doi: 10.1111/j.1755-0998.2010.02924.x

Leme J.L.M. 1973. Anatomy and systematics of the Neotropical Strophocheiloidea (Gastropoda, Pulmonata) with the description of a new family. Arquivos de Zoologia 23:295-337.

Ramírez R. \& S. Cáceres. 1991. Caracoles terrestres (Mollusca, Gastropoda) comestibles en el Perú. Boletín de Lima (Peru). (Set 1991) 67-74.

Ramírez, R.; C. Paredes y J. Arenas. 2003. Moluscos del Perú. Revista de Biología Tropical 51:225-284.

Rohlf F.J. \& D. Slice. 1990. Extensions of the Procrustes Method for the Optimal Superimposition of Landmarks. Systematic Zoology 39:40-59. doi: 10.2307/2992207

Rohlf F.J. 2010. tpsDig, version 2.16. Stony Brook University.

Schilthuizen M. \& M. Haase. 2010. Disentangling true shape differences and experimenter bias: are dextral and sinistral snail shells exact mirror images? Journal of Zoology 282:191-200. doi:10.1111/j.1469-7998.2010.00729.x.

Simone L.R.L. 2006. Land and Freshwater Molluscs of Brazil. FAPESP/EGB. 390 pp.

Smith U.E. \& J.R. Hendricks. 2013. Geometric Morphometric Character Suites as Phylogenetic Data: Extracting Phylogenetic Signal from Gastropod Shells. Systematic Biology 62:366385. doi: $10.1093 /$ sysbio/syt002.

Suvorov N.A. 2002. Prospects for Studies of Morphological Variability of Land Pulmonate Snails. Biology Bulletin of the Russian Academy of Sciences 29:455-467. 